\title{
Traffic Grooming Based on Shortest Path in Optical WDM Mesh Networks`
}

\author{
Yeo-Ran Yoon, Tae-Jin Lee, Min Young Chung, and Hyunseung Choo \\ Lambda Networking Center, \\ School of Information and Communication Engineering, \\ Sungkyunkwan University, Korea \\ \{ookuma, tjlee, mychung, choo\}@ece.skku.ac.kr
}

\begin{abstract}
This paper investigates the static traffic grooming in WDM optical mesh networks. Our objective is to improve the network throughput and to minimize the blocking probability. As take care of this problem efficiently, we propose Shortest-path First Traffic grooming(SFT) algorithm. The comprehensive computer simulation shows that our proposed algorithm is up to about $14 \%$ superior to the existing one known to be effective.
\end{abstract}

\section{Introduction}

Optical wavelength-division multiplexing (WDM) is a promising technology to accommodate the explosive growth of Internet and telecommunication traffics. With each optical link capable of carrying traffic on several wavelengths, each one of which supports traffic in the Gbps range. However, the traffic requested by individual connection is still in the Mbps range. Hence, to utilize the available bandwidth efficiently, several connections have to be grouped onto the same wavelength.This requires strategic routing and wavelength assignment (RWA) of each connection 1 .

The problem of RWA on sub-wavelength demands with the objectives of minimizing the network cost and optimizing network throughput is called "traffic grooming" problem which refers to the techniques used to combine lower-capacity components onto available wavelengths. Traffic grooming has received considerable attention recently, and there are many related work in the literature. In recent past, there have been efforts towards solving the traffic grooming problem for mesh networks. This issue has been addressed in both the static [4,5] as well as the dynamic 3 scenarios.

In this paper, we consider the static traffic grooming for the WDM mesh networks. We propose a Shortest-path First Traffic grooming (SFT) algorithm in objective to maximize the network throughput and to minimize the blocking probability. The proposed algorithm uses effective routing method instead of previous shortest path routing, then low-capacity connection requests are efficiently

\footnotetext{
* This work was supported in parts by Brain Korea 21 and the Ministry of Information and Communication, Korea. Corresponding author: Prof. H. Choo.
} 
groomed together and carried. According to the computer simulation, the SFT algorithm achieves $14 \%$ improved performance in terms of the network throughput, compared with the Maximizing Single-hop traffic(MST) algorithm [5].

\section{Related Works and Problem Statement}

Current and future WDM networks are increasingly arranged in general mesh topologies. Indeed, much recent work has focused on grooming traffic in mesh networks [3,4,5. Existing work on traffic grooming has considered two types of traffic model: static traffic model and dynamic random traffic one. In the static traffic model, all capacity demands are known in advance and do not change over time. The studies for static grooming are [4,5. In the dynamic random traffic model, a demand is assumed to arrive at a random time and last for a certain amount of time in random. The work in [3] studied traffic grooming issues in dynamic traffic environment.

In general when solving the traffic grooming problem, traffic requests are carried through single-hop grooming and multi-hop grooming. Single-hop grooming is a method that allows a connection to transverse a single lightpath. On the other hand, multi-hop grooming is a method that allows a connection to traverse multiple lightpaths. In multi-hop grooming, a connection can be dropped at intermediate nodes and groomed with other low-capacity connections on different lightpaths before it reaches its destination node.

The traffic grooming problem can be formulated as follows [5]. Given a network configuration (including physical topology, where each edge is a physical link, number of transceivers at each node, number of wavelengths on each fiber, and the capacity of each wavelength) and a set of connection requests with different bandwidth granularities, such as OC-12, OC-48, etc., we need to determine how to set up lightpaths to satisfy the connection requests. Because of the subwavelength granularity of the connection requests, one or more connections can be multiplexed on the same lightpath.

\section{Shortest Path First Traffic Grooming (SFT)}

Our goal is to improve the network throughput by using the efficient routing algorithm for traffic grooming. MST algorithm [5] assigns lightpaths by adaptive routing using Dijkstra's shortest-path, however it finds the shortest path under the current network status which may not be the real shortest one because the network status is changed frequently. For that reason, we apply a routing algorithm that preferentially assigns the shortest paths which are found on the original network status in the proposed SFT algorithm.

The proposed SFT algorithm uses both fixed routing based on shortest paths and adaptive routing. Firstly this algorithm only assigns lightpaths of connection requests which are available to use shortest paths in the original network status. Then connection requests which are not proper to use shortest paths go for employing adaptive routing before trying other wavelengths. If we use SFT 
algorithm employing this routing method, more demands are carried through shortest paths and resources are used efficiently than that of using MST. Furthermore, connection requests which are not carried through single-hop traffic grooming have more chances to be carried through multi-hop grooming.

Step 1: Constructing a virtual topology

1.1: Sort all node pairs $\left(s_{i}, d_{i}\right)$ according to the sum of uncarried traffic requests $T\left(s_{i}, d_{i}\right)$ between $s_{i}$ and $d_{i}$, and put them into a list $L$ in a descending order.

1.2: Find a shortest path for each $\left(s_{i}, d_{i}\right)$ and its hop count $\left(h\left(s_{i}, d_{i}\right)\right)$ by using Dijkstra's shortest-path algorithm.

1.3: Setup a lightpath for each node pair $\left(s_{i}, d_{i}\right)$ in the list $L$ just by using the shortest path obtained in Step $\mathbf{1 . 2}$ subject to transceiver constraints. If we assign the lightpath, let $T\left(s_{i}, d_{i}\right)=\operatorname{Max}\left[T\left(s_{i}, d_{i}\right)-C, 0\right]$ and delete edges of the assigned lightpath from the physical topology. If we cannot use the shortest path obtained in Step 1.2, send $\left(s_{i}, d_{i}\right)$ to list $L^{\prime}$. And if there are not available lightpaths due to short of transceivers or deleted edges, move $\left(s_{i}, d_{i}\right)$ to $L^{\prime \prime}$.

1.4: Setup lightpaths for node pairs $\left(s_{i}, d_{i}\right) \mathrm{s}$ in $L^{\prime}$ using the adaptive routing, subject to transceiver constraints. If we fail, move $\left(s_{i}, d_{i}\right)$ to $L^{\prime \prime}$;

Otherwise, we assign the lightpath and let $\mathrm{T}\left(s_{i}, d_{i}\right)=\operatorname{Max}\left[T\left(s_{i}, d_{i}\right)-C\right.$, $0]$. Then edges of the assigned lightpath are deleted from the physical topology.

1.5: Go to Step 1.3 until lists $L$ and $L^{\prime}$ become empty.

Step 2: Routing the low-speed connections on the virtual topology constructed in Step 1.

2.1: Route all connection requests which can be carried through single lightpath hop, and update the network status for the virtual topology.

2.2: Route the remaining connection requests using currently available spare capacity of the virtual topology based on their sum of uncarried traffic request value $T\left(s_{i}, d_{i}\right)$.

Step 3: Back to the original network status, store $L^{\prime \prime}$ in $L$ and repeat Steps 1 and $\mathbf{2}$ until entire wavelengths are fully exhausted

\section{Numerical Results}

The performance of SFT algorithm developed in this paper is compared against well-known MST on the NSFNET and random network topologies. NSFNET has 14 nodes and 21 links as shown in Fig. 1(a) and random networks are generated based on 2. We compare SFT and MST algorithms in terms of network throughput. We show the results of the MST and the proposed SFT algorithm on NSFNET in Fig. 1(b). We have constructed the resulting graphs for number of wavelengths per each fiber link versus network throughput. The results show that our SFT performs better than MST algorithm with respect to network throughput at any number of wavelengths per link. As you see here, the SFT algorithm outperforms 3\% 14\% over MST. 


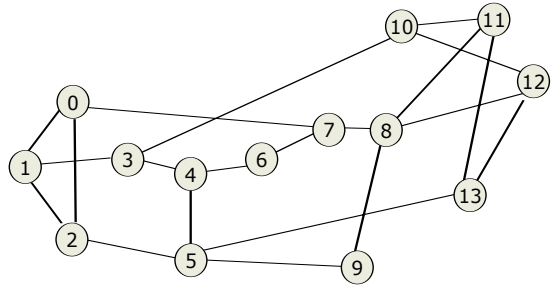

(a) NSFNET topology.

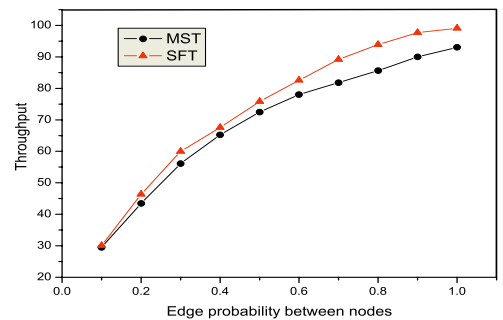

(c) Throughput in random networks $\quad\left(N=15, W=10, T_{r}\right.$ $=15)$.

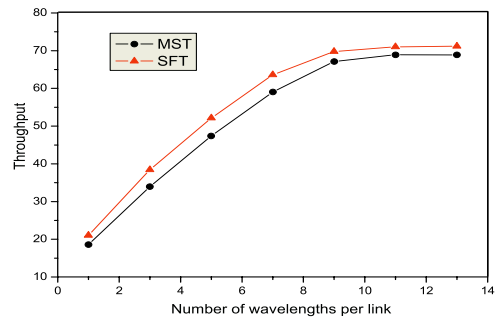

(b) Throughput comparison in NSFNET $\quad\left(T_{r}=15\right)$.

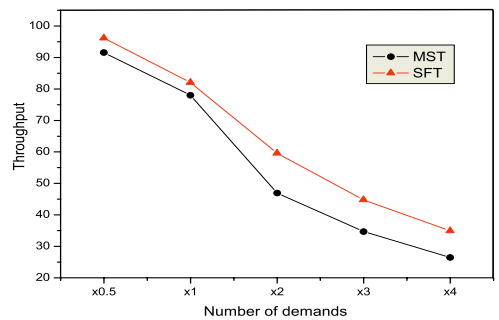

(d) Throughput in random networks $\quad\left(N=15, P_{e}=0.6, W\right.$ $\left.=10, T_{r}=15\right)$.

Fig. 1. Throughput comparison

In Fig. 1(c), we compare the network throughput of MST and SFT algorithms in random topologies with 15 nodes when the edge existence probability between node pairs $\left(P_{e}\right)$ increases. We observe that the SFT algorithm demonstrates $4 \%$ 10\% higher throughput than the MST. In Fig. 1(d), we plot the results that the number of demands versus network throughput in random topologies. We can see that the throughput of SFT algorithm acquires $5 \% \sim 18 \%$ better performance than that of MST. And as the number of demands increases, the performance of the SFT algorithm becomes better.

\section{Conclusion}

We investigated the traffic grooming in WDM optical networks and proposed an improved heuristic algorithm with respect to network throughput called Shortest path First Traffic grooming (SFT). This algorithm consider the shortest path on original graph and uses as many shortest paths as possible for the demands. We showed that the SFT algorithm outperforms MST with various aspects in NSFNET and random networks. 


\section{References}

1. R. Ramaswami and K. N. Sivarajan, Optical Networks: A Practical Perspective. San Francisco, CA: Morgan Kaufmann, 1998.

2. A.S. Rodionov and H. Choo, "On generating random network structures: Connected Graphs," Springer-Verlag Lecture Notes in Computer Science, vol. 3090, pp. 483 491, Sep. 2004.

3. S. Thiagarajan and A. K. Somani, "Capacity fairness of WDMnetworks with grooming capability," Opt. Networks Mag., vol. 2, no. 3, pp. 24-31, May/June 2001.

4. H. Zhu, H. Zang, K. Zhu, and B. Mukherjee, "A novel, generic graph model for traffic grooming in heterogeneous WDM mesh networks," IEEE/ACM Trans. Networking, vol. 11, pp. 285-299, Apr. 2003.

5. K. Zhu and B. Mukherjee, "Traffic grooming in an optical WDM mesh network," IEEE J. Select. Areas Commun., vol. 20, pp. 122-133, Jan. 2002. 